\title{
Maximizing of Asymptomatic Stage of Fast Progressive HIV Infected Patient Using Embedding Method
}

\author{
Hassan Zarei, Ali Vahidian Kamyad, Sohrab Effati \\ Department of Applied Mathematics, Ferdowsi University of Mashhad, Mashhad, Iran \\ E-mail: zarei2003@yahoo.com \\ Received July 7, 2010; revised July 15, 2010; accepted July 23, 2010
}

\begin{abstract}
A system of ordinary differential equations, which describe various aspects of the interaction of HIV with healthy cells in fast progressive patient, is utilized, and an optimal control problem is constructed to prolong survival and delay the progression to AIDS as far as possible, subject to drug costs. Optimal control problem is approximated by linear programming model using measure theoretical approach and suboptimal combinations of reverse transcriptase inhibitor (RTI) and protease inhibitor (PI) drug efficacies are proposed. The Comparison of healthy CD4+ T-cells counts, virus particles and immune response, before and after the treatment is introduced.
\end{abstract}

Keywords: HIV Model, Optimal Control, Measure Theory, Linear Programming

\section{Introduction}

Human Immunodeficiency Virus infects CD4+ T-cells, which are an important part of the human immune system, and other target cells. The infected cells produce a large number of viruses. Medical treatments for HIV have greatly improved during the last two decades. Highly active antiretroviral therapy (HAART) allows for the effective suppression of HIV-infected individuals and prolongs the time before the onset of Acquired Immune Deficiency Syndrome (AIDS) for years or even decades and increase life expectancy and quality to the patient but antiretroviral therapy cannot eradicate HIV from infected patients because of long-lived infected cells and sites within the body where drugs may not achieve effective levels [1-3]. HAART contain two major types of anti-HIV drugs, reverse transcriptase inhibitors (RTI) and protease inhibitors (PI). Reverse transcriptase inhibitors prevent HIV from infecting cells by blocking the integration of the HIV viral code into the host cell genome. Protease inhibitors prevent infected cells from replication of infectious virus particles, and can reduce and maintain viral load below the limit of detection in many patients. Moreover, treatment with either type of drug can also increase the CD4+ T-cell count that are target cells for HIV.

Many of the host-pathogen interaction mechanisms during HIV infection and progression to AIDS are still unknown. Mathematical modeling of HIV infection is of interest to the medical community as no adequate animal models exist in which to test efficacy of drug regimes. These models can test different assumptions and provide new insights into questions that is difficult to answer by clinical or experimental studies. A number of mathematical models have been formulated to describe various aspects of the interaction of HIV with healthy cells, See [4]. The basic model of HIV infection is presented by Perelson et al. [5] that contain three state variables healthy CD4+ T-cells, infected CD4+ T-cells and concentration of free virus. Another model is presented in [6] that although maintaining a simple structure, the model offers important theoretical insights into immune control of the virus based on treatment strategies Furthermore this modified model is developed to describe the natural evolution of HIV infection, as qualitatively described in several clinical studies [7].

Some authors use mathematical model for HIV infection in conjunction with control theory to achieve appropriate goals, by incorporating the effects of therapy on an HIV-infected individuals. For example, these goals my be maximizing the level of healthy $\mathrm{CD} 4+\mathrm{T}$-cells and minimizing the cost of treatment [8-12], maximizing immune response and minimizing both the cost of treatment and viral load [13,14], maximizing both the level of healthy CD4+ T-cells and immune response and minimizing the cost of treatment [15], Maximizing the level of healthy CD4+ T-cells while minimizing both the side effects and drug resistance [16] and maximizing survival 
time of patient subject to drug cost [17] and etc.

The papers [18-21] consider only RTI medication while the papers [22,23] consider only PIs. In [24-27] all effects of a HAART medication are combined to one control variable in the model. In [13,28-31] dynamical multidrug therapies based on RTIs and PIs are designed.

In this paper, we consider a mathematical model of HIV dynamics that includes the effect of antiretroviral therapy, and perform an analysis of optimal control regarding appropriate goal.

The paper is organized as follows. In Section 2, the underlying HIV mathematical model is described. Our formulation of the control problem which attempts to delay appearance of AIDS as far as possible is described in Section 3. Formulated optimal control problem is approximated by linear programming (LP) problem. Related procedure is described in Section 4. Numerical results obtained from using LP model are presented in Section 5. Finally Section 6 is assigned to concluding remarks.

\section{Presentation of a Working Model}

In this paper, the pathogenesis of HIV is modeled with a system of ordinary differential equations (ODEs) described in [7]. This model can be viewed as an extension of basic HIV Models of Perelson et al. [5].

$$
\begin{gathered}
\dot{x}=\lambda-d x-r x v \\
\dot{y}=r x v-a y-\rho y z \\
\dot{w}=c x y w-q y w-b w \\
\dot{z}=q y w-h z \\
\dot{v}=k\left(1-u_{P}\right) y-\tau v \\
\dot{r}=r_{0}-u_{R}
\end{gathered}
$$

Most of the terms in the model have straightforward interpretations as following:

The first equation represents the dynamics of the concentration of healthy CD4+ T-cells $(x)$. The healthy CD4+ T-cells are produced from a source, such as the thymus, at a constant rate $\lambda$, and die at a rate $d x$. The cells are infected by the virus at a rate $r x v$. The second equation describes the dynamics of the concentration of infected CD4+ T-cells (y). The infected CD4+ T-cells result from the infection of healthy CD4+ T-cells and die at a rate $a y$ and killed by cytotoxic T-lymphocyte effectors CTLe $(z)$ at a rate $\rho y z$. The population of CTLs is subdivided into precursors or CTLp $(w)$, and effectors or CTLe (z). Equations (3)-(4) describe the dynamics of these compartments. In accordance with experimental findings [32] establishment of a lasting CTL response depends on CD4+ T-cell help, and that HIV impairs T helper cell function. Thus, proliferation of the CTLp population is given by cxyw and is proportional to both virus load $(y)$ and the number of uninfected $\mathrm{T}$ helper cells $(x)$. CTLp differentiation into effectors occurs at a rate cqyw. Finally, CTLe die at a rate $h z$. Equation (5) describes the dynamics of the free-virus particles $(v)$. These free-virus particles are produced from infected CD4+ T-cells at a rate $k y$ and are cleared at a rate $\tau v$. Model also contain an index of the intrinsic virulence or aggressiveness of the virus $(r)$. This index increases linearly in the case of an untreated HIV-infected individual, with a growth rate that depends on the constant $r_{0}$ Finally Equation (6) describes the dynamic of this index. In model variables $u_{P}$ and $u_{R}$ denotes protease inhibitors (PI) and reverse transcriptase inhibitors (RTI), respectively. $u_{R}$ reduces infection rate of healthy CD4+ T-cells by reducing the growth rate of the aggressiveness of the virus $(r)$ and $u_{P}$ prevents virus production by reducing the production rate from infected CD4+ T-cells.

The model has several parameters that must be assigned for numerical simulations. The descriptions, numerical values and units of the parameters are summarized in Table 1. These descriptions and values were taken from [7]. We note that Equations (1)-(6) with these parameters, model dynamics of fast progressive patients (FPP).

\section{Optimal Control Formulation}

In clinical practice, Anti-retroviral therapy is initiated at $t_{0}$, the time at which CD4+ T-cell counts reach 350 cells $/ \mu$. The transition from HIV to AIDS occurred when patients CD4+ T-cell count falls below $C D 4_{\text {AIDS }}^{+}$ around 200 cells $/ \mu \mathrm{l}$. Our aim is to propose drug regimen

Table 1. Parameter Values for the HIV model.

\begin{tabular}{cll}
\hline Parameters & \multicolumn{1}{c}{ Value/Unit } & \multicolumn{1}{c}{ Description } \\
\hline$\lambda$ & $7{\text { cells } \mu l^{-1} \text { day }^{-1}}^{-1}$ & Healthy CD4+ Production \\
$d$ & $7 \times 10^{-3}$ day $^{-1}$ & Healthy CD4+ clearance \\
$a$ & 0.0999 day $^{-1}$ & Infected CD4+ clearance \\
$\rho$ & $2 \mu$ ceells $^{-1}$ day $^{-1}$ & Infected CD4+ kill \\
$c$ & $5 \times 10^{-6} \mu^{2}$ cells $^{-2}$ day $^{-1}$ & CTLp proliferation \\
$q$ & $6 \times 10^{-4} \mu_{\text {lcells }}{ }^{-1}$ day $^{-1}$ & CTLp differentiation \\
$b$ & 0.017 day $^{-1}$ & CTLp clearance \\
$h$ & 0.06 day $^{-1}$ & CTLe clearance \\
$k$ & 300 copiesml $^{-1}$ cells $^{-1} \mu$ lday $^{-1}$ & Virus production \\
$\tau$ & 0.2 day $^{-1}$ & Virus clearance \\
$r_{0}$ & $10^{-9}$ copies $^{-1}$ ml day $^{-2}$ & Virulence growth
\end{tabular}


to maximize asymptomatic stage time or equivalently prolong survival and delays the progression to AIDS as far as possible, subject to drug costs. This can be modeled as follows:

Assume that the onset of AIDS occurs after time $t_{f}$. Hence we should have:

$$
x\left(t_{f}\right)=C D 4_{A I D S}^{+}, x(t) \geq C D 4_{A I D S}^{+}, \forall t \in\left[t_{0}, t_{f}\right]
$$

We follow [8] and [22] in assuming systemic costs of the PI and RTI drugs treatment is proportional to $u_{P}^{2}(t)$ and $u_{R}^{2}(t)$ at time $t$ respectively. Therefore Overall cost of the PI and RTI drugs treatment is $\int_{t_{0}}^{t_{f}} u_{P}^{2}(t) d t$ and $\int_{t_{0}}^{t_{f}} u_{R}^{2}(t) d t$ respectively and overall cost of treatment is given by $\int_{t_{0}}^{t_{f}} u_{P}^{2}(t) d t+\sigma \int_{t_{0}}^{t_{f}} u_{R}^{2}(t) d t$. Because symmetric costs for two types of drugs are in different scale, coefficient $\sigma$ is set to balance them. Administration of drugs in high dose, are toxic to the human body. Moreover emergence of drug resistant strains is one of the basic complications in drug treatments. Many authors have ignored drug resistance issues, since fixing a maximum cost for a drug regime is equivalent to only administering a limited amount of chemotherapeutic agent. If that limited amount is chosen to be sufficiently small positive $\gamma$, the risk of drug resistance can be largely ignored. Therefore we impose following constraint on drug cost:

$$
\int_{t_{0}}^{t_{f}}\left(u_{P}^{2}(t)+\sigma u_{R}^{2}(t)\right) d t \leq \gamma
$$

Setting, $\xi=(x, y, w, z, v, r)$ and $u(t)=\left(u_{P}, u_{R}\right)$ the differential Equations (1)-(6) can be represented in a generalized form as:

$$
\dot{\xi}(t)=g(t, \xi(t), u(t))=\left(\begin{array}{c}
\lambda-d \xi_{1}-\xi_{1} \xi_{5} \xi_{6} \\
\xi_{1} \xi_{5} \xi_{6}-a \xi_{2}-\rho \xi_{2} \xi_{4} \\
c \xi_{1} \xi_{2} \xi_{3}-q \xi_{2} \xi_{3}-b \xi_{3} \\
q \xi_{2} \xi_{3}-h \xi_{4} \\
k\left(1-u_{P}\right) \xi_{2}-\tau \xi_{5} \\
r_{0}-u_{R}
\end{array}\right)
$$

Now with respect to above descriptions and (7) and (8) the optimal drug regime problem can be stated as follows:

$$
\max _{u, t_{f}} \int_{t_{0}}^{t_{f}} d t
$$

subject to

$$
\dot{\xi}=g(t, \xi, u)
$$

$$
\begin{aligned}
& \int_{t_{0}}^{t_{f}}\left(u_{P}^{2}(t)+\sigma u_{R}^{2}(t)\right) d t \leq \gamma \\
& \xi_{1}\left(t_{0}\right)=\xi_{0}, \quad \xi_{1}\left(t_{f}\right)=C D 4_{A I D S}^{+} \\
& \xi_{1}(t) \geq C D 4_{A I D S}^{+}, \quad t \in\left[t_{0}, t_{f}\right]
\end{aligned}
$$

We refer to this time optimal control problem as TOCP. Some problems may arise in the quest for the optimal solution. For example, may not exist control function $u($.$) and corresponding state \xi($.$) and final$ time $t_{f}$ that satisfy in (11)-(14). In order to overcome these difficulties in the next section we transfer the TOCP into a modified problem in measure space.

\section{Approximation of TOCP by Linear Programming Model}

Using the measure theory for solving optimal control problems based on the idea of Young [33], which was applied for the first time by Wilson and Rubio [34], has been theoretically established by Rubio in [35]. Then the method has been extended and improved by Mehne et al. [36] for solving time optimal control problems that leads to approximation of problem by linear programming (LP) model. We shall follow their approach here.

\subsection{Transformation to Functional Space}

We assume that state variable $\xi($.$) and control input$ $u\left(\right.$.), get their values in the compact sets $A=A_{1} \times \cdots \times$ $A_{6} \subset \mathfrak{R}^{6}$ and $U=U_{1} \times U_{2} \subset \mathfrak{R}^{2}$, respectively. Set $J=\left[t_{0}, t_{f}\right]$.

Definition 4.1.1. We define a triple $p=\left[t_{f}, \xi, u\right]$ to be admissible if the following conditions hold:

1) The vector function $\xi($.$) be absolutely continuous$ and belongs to $A$ for all $t \in J$.

2) The function $u($.$) takes its value in the set U$ and is Lebesgue measurable on $J$.

3) $p$ satisfies in the system (11)-(14), i.e. on $J^{0}$, the interior set of $J$.

We assume that the set of all admissible triples is nonempty and denote it by $W$. Let $p$ be an admissible triple and $B$ be an open ball in $\Re^{6}$ containing $J \times A$ and $C^{\prime}(B)$ be the space of all real-valued continuous differentiable functions on it. Let $\varphi \in C^{\prime}(B)$ and define $\varphi^{g}$ as follows: 


$$
\begin{aligned}
& \varphi^{g}(t, \xi(t), u(t))=\frac{d \varphi(t, \xi(t))}{d t} \\
& =\sum_{j=1}^{6} \frac{\partial \varphi(t, \xi(t))}{\partial \xi_{j}} g_{j}(t, \xi(t), u(t))+\frac{\partial \varphi(t, \xi(t))}{\partial t}
\end{aligned}
$$

for each $[t, \xi(t), u(t)] \in \Omega$, where $\Omega=J \times A \times U$. The function $\varphi^{g}$ is in the space $C(\Omega)$, the set of all continuous functions on the compact set $\Omega$. Since $p=$ $\left[t_{f}, \xi, u\right]$ is an admissible triple, we have

$$
\begin{aligned}
& \int_{t_{0}}^{t_{f}} \varphi^{g}(t, \xi(t), u(t)) d t \\
& =\varphi\left(t_{f}, \xi\left(t_{f}\right)\right)-\varphi\left(t_{0}, \xi\left(t_{0}\right)\right)=\Delta \varphi
\end{aligned}
$$

for all $\varphi \in C^{\prime}(B)$. Let $D\left(J^{0}\right)$ be the space of infinitely differentiable all real-valued function with compact support in $J^{0}$. Define:

$$
\begin{aligned}
& \psi^{j}(t, \xi(t), u(t))=\xi_{j}(t) \psi^{\prime}(t)+g_{j}(t, \xi(t), u(t)) \psi(t), \\
& j=1, \ldots, 6 \forall \psi \in D\left(J^{0}\right)
\end{aligned}
$$

Then if $p=\left[t_{f}, \xi, u\right]$ be an admissible triple for $j=1, \ldots, 6$, and $\forall \psi \in D\left(J^{0}\right)$, from (17) we have

$$
\begin{aligned}
& \int_{t_{0}}^{t_{f}} \psi^{j}(t, \xi(t), u(t)) d t=\int_{t_{0}}^{t_{f}} \xi_{j}(t) \psi^{\prime}(t) d t \\
& +\int_{t_{0}}^{t_{f}} g_{j}(t, \xi(t), u(t)) \psi(t) d t=\left.\xi_{j}(t) \psi(t)\right|_{t_{0}} ^{t_{f}} \\
& +\int_{t_{0}}^{t_{f}}\left\{g_{j}(t, \xi(t), u(t))-\dot{\xi}_{j}(t)\right\} \psi(t) d t
\end{aligned}
$$

since the function $\psi($.$) has compact support in J^{0}$, so

$$
\begin{aligned}
& \psi\left(t_{0}\right)=\psi\left(t_{f}\right)=0 \text { and } \dot{\xi}_{j}=g_{j} \text { so } \\
& \int_{t_{0}}^{t_{f}} \psi^{j}(t, \xi(t), u(t)) d t=0
\end{aligned}
$$

Also by choosing the functions which are dependent only on time, we have:

$$
\int_{t_{0}}^{t_{f}} \vartheta(t, \xi(t), u(t)) d t=a_{\vartheta}, \forall \vartheta \in C^{1}(\Omega)
$$

where $C^{1}(\Omega)$ is the space of all functions in $C(\Omega)$ that depend only on time and $a_{\vartheta}$ is the integral of $\vartheta$ on $J$. Equations (16), (18) and (19) are really weak form of (11), (13) and (14). We note that, the role of constraint (13) is considered on the right side of equation (16) by considering functions $\varphi \in C^{\prime}(B)$ which are monomials of $\xi_{1}$. Furthermore, the constraint (14) is considered, by choosing appropriate set $A$. Now we consider the following positive linear functional on $C(\Omega)$.

$$
\Gamma_{p}: F \rightarrow \int_{J} F(t, \xi(t), u(t)) d t, \forall F \in C(\Omega)
$$

Proposition 4.1.1. Transformation $p \rightarrow \Gamma_{p}$ of admissible triples in $W$ into the linear mappings $\Gamma_{p}$ defined in (20) is an injection.

Proof. We must show that if $p_{1} \neq p_{2}$, then $\Gamma_{p_{1}} \neq \Gamma_{p_{2}}$. Let $p_{j}=\left[t_{f_{j}}, \xi_{j}, u_{j}\right], j=1,2$ be different admissible triples. If $t_{f_{1}}=t_{f_{2}}$, then there is a subinterval of $\left[t_{0}, t_{f_{1}}\right]$, say $J_{1}$, where $\xi_{1}(t) \neq \xi_{2}(t)$ for each $t \in J_{1}$. A continuous function $F$ can be constructed on $\Omega$ so that the right-hand side of (20) corresponding to $p_{1}$ and $p_{2}$ are not equal. For instance, assume $F$ is independent of $u$ such that for all $t \in J_{1}$, this function is positive on the appropriate portion of the graph of $\xi_{1}(t)$, and zero on $\xi_{2}(t)$, then the linear functional are not equal. In other word if $t_{f_{1}} \neq t_{f_{2}}$, then $\Gamma_{p_{1}}$ and $\Gamma_{p_{2}}$ have different domains and are not equal.

Thus, the TOCP (10)-(14) is converted to following optimization problem in functional space:

$$
\underset{\Gamma_{p}}{\operatorname{Maximize}} \Gamma_{p}(1) \quad \text { (from (10)) }
$$

Subject to

$$
\begin{gathered}
\Gamma_{p}\left(\phi^{g}\right)=\Delta \phi, \phi \in C^{\prime}(B) \quad(\text { from (16)) } \\
\Gamma_{p}\left(\psi^{j}\right)=0, j=1, \ldots, 6, \psi \in D\left(J^{0}\right) \quad(\text { from (18)) } \\
\Gamma_{p}(\vartheta)=a_{\vartheta}, \vartheta \in C^{1}(\Omega) \quad(\text { from (19)) } \\
\Gamma_{p}(H) \leq \gamma \quad(\text { from (12)) }
\end{gathered}
$$

where $H(t, \xi(t), u(t))=u_{p}^{2}(t)+\sigma u_{R}^{2}(t)$.

\subsection{Transformation to Measure Space}

Let $M^{+}(\Omega)$ denotes the space of all positive Radon measures on $\Omega$. By the Riesz representation theorem, there exists a unique positive Radon measure $\mu$ on $\Omega$ such that:

$$
\begin{aligned}
& \Gamma_{p}(F)=\int_{J} F(t, \xi(t), u(t)) d t \\
& =\int_{\Omega} F(t, \xi, u) d \mu \equiv \mu(F), \quad F \in C(\Omega)
\end{aligned}
$$

So, we may change the space of optimization problem 
to measure spaces. In other words, the optimization problem in functional space (21)-(25) can be replaced by the following new problem in measure space:

$$
\underset{\mu \in M^{+}(\Omega)}{\operatorname{Maximize}} \mu(1)
$$

Subject to

$$
\begin{gathered}
\mu\left(\varphi^{g}\right)=\Delta \varphi, \varphi \in C^{\prime}(B) \\
\mu\left(\psi^{j}\right)=0, \quad j=1, \ldots, 6, \psi \in D\left(J^{0}\right) \\
\mu(\vartheta)=a_{\vartheta}, \vartheta \in C^{1}(\Omega) \\
\mu(H) \leq \gamma
\end{gathered}
$$

We shall consider the maximization of (27) over the set $Q$ of all positive Radon measures on $\Omega$ satisfying (28)-(31). The main advantages of considering this measure theoretic from of the problem is the existence of an optimal measure in the set $Q$ which this point can be studied in a straightforward manner without having to impose conditions such as convexity which may be artificial.

Theorem 4.2.1. The measure theoretical problem of maximizing (27) with equality and inequality constraints (28)-(31) has an optimal solution $\mu^{*}$.

Proof. As we will show in the next, (29) and (30) are special version of (28). Therefore, the set $Q$ can be written as $Q=Q_{1} \cap Q_{2}$ where

$$
Q_{1}=\bigcap_{\varphi \in C^{\prime}(B)}\left\{\mu \in M^{+}(\Omega): \mu\left(\varphi^{g}\right)=\Delta \phi\right\}
$$

and

$$
Q_{2}=\left\{\mu \in M^{+}(\Omega): \mu(H) \leq \gamma\right\} .
$$

Assume that $p=\left[t_{f}, \xi, u\right]$ is an admissible triple. It is well known that, the set $\left\{\mu \in M^{+}(\Omega): \mu(1)=t_{f}-t_{0}\right\}$ is compact in weak ${ }^{*}$-topology. Furthermore, $Q_{1}$ as intersection of inverse image of closed singleton sets $\{\Delta \varphi\}$ under continuous functions $\mu \rightarrow \mu\left(\varphi^{g}\right)$ is also closed. It can be shown in a similar way that $Q_{2}$ is closed. Thus, $Q$ is a closed subset of a compact set. This proves the compactness of the set $Q$. Since the functional $\mu \rightarrow \mu(1)$ mapping the compact set $Q$ on the real line, is continuous and so has a maximum on the compact set $Q$.

Next, based on analysis in [35], the problem (27)-(31) is approximated by a LP problem and a triple $p^{*}$ which approximate the action of $\mu^{*} \in Q$ is achieved.

\subsection{Approximation}

Problem (27)-(31) is an infinite dimensional linear programming problem and all the functions in (28)-(31) are linear with respect to measure $\mu$. Of course, it is an infinite dimensional LP problem, because $M^{+}(\Omega)$ is infinite dimensional space. It is possible to approximate the solution of this problem by the solution of a finitedimensional LP of sufficiently large dimension. Also, from the solution of this new finite dimensional LP we induce an approximated admissible triple in a suitable manner. We shall first develop an intermediate problem, still infinite-dimensional by considering the maximization (27), not over the set $Q$ but over a subset of $M^{+}(\Omega)$ with only a finite numbers of the constraints in (28)-(31) being satisfied. This will be achieved by choosing countable sets of functions whose linear combinations are dense in the sets $C^{\prime}(B), C^{1}(\Omega)$ and $D\left(J^{0}\right)$, and then selecting a finite number of them. Assume the set $\left\{\varphi_{i}: i=1,2, \ldots\right\}$ be such that the linear combinations of the functions $\varphi_{i} \in C^{\prime}(B)$ are uniformly dense in $C^{\prime}(B)$. For instance, these functions can be taken to be monomials in $t$ and the components of the vector $\xi$. As we will show in the next, some of these monomials are suitable for our problem and are as follows:

$$
\begin{aligned}
& t^{i} \xi_{1}^{j} \text { and } \xi_{1}^{j} \xi_{h}^{i}, i \in\{0,1\}, j \in\{1,2, \ldots\}, \\
& h \in\{2,3,4,5,6\}
\end{aligned}
$$

Let set $\left\{\psi_{i}: i=1,2, \ldots\right\}$ be such that linear combinations of the functions $\psi_{i} \in D\left(J^{0}\right)$ are uniformly dense in $D\left(J^{0}\right)$. For $r=1,2, \ldots$ some of these functions can be taken as follows [36]:

$$
\psi_{2 r-1}(t)=\left\{\begin{array}{cc}
\sin \left(\frac{2 \pi r\left(t-t_{0}\right)}{\Delta T}\right) & t \leq t_{l} \\
0 & \text { otherwise }
\end{array}\right.
$$

and

$$
\psi_{2 r}(t)=\left\{\begin{array}{cc}
1-\cos \left(\frac{2 \pi r\left(t-t_{0}\right)}{\Delta T}\right) & t \leq t_{l} \\
0 & \text { otherewise }
\end{array}\right.
$$

where $\Delta T=t_{l}-t_{0}$ and $t_{l}$ is a lower bound for optimal time which can be obtained using controllability.

Finally, let set $\left\{\vartheta_{i}: i=1,2, \ldots\right\}$ be such that linear combinations of the functions $\vartheta_{i} \in C^{1}(\Omega)$ are uni- 
formly dense in $C^{1}(\Omega)$. These functions can be considered monomials in $t$ as follows:

$$
\vartheta_{s}(t)=t^{s}, \quad s=0,1,2 \ldots
$$

Remark 1. With respect to (15) and (17) it can be seen that (29) and (30) are also achieved from (28) by setting $\varphi(t, \xi(t))=\xi_{j}(t) \psi(t)$ and $\varphi(t, \xi(t))=\int_{0}^{t} \vartheta(\tau) d \tau$ respectively.

The first approximation will be completed by using above subjects and the following propositions.

Proposition 4.3.1. Consider the linear program consisting of the maximizing function $\mu \rightarrow \mu(1)$ over the set $Q_{M}$ of measures in $M^{+}(\Omega)$ satisfying:

$$
\begin{gathered}
\mu\left(\varphi_{i}^{g}\right)=\Delta \varphi_{i}, \quad i=1, \ldots, M \\
\mu(H) \leq \gamma
\end{gathered}
$$

Then $\lambda_{M} \equiv \max _{Q_{M}} \mu(1)$ tends to $\lambda \equiv \max _{Q} \mu(1)$ as $M \rightarrow \infty$.

Proof. We have $Q_{1} \supseteq Q_{2} \supseteq \cdots \supseteq Q_{M} \supseteq \cdots \supseteq Q$; hence, $\lambda_{1} \geq \lambda_{2} \geq \cdots \geq \lambda_{M} \geq \cdots \geq \lambda$. Therefore, $\left\{\lambda_{n}\right\}$ is non increasing and bounded sequence then converges to a number $\zeta$ such that $\zeta \geq \lambda$. We show that, $\zeta=\lambda$.

Set $R \equiv \bigcap_{M=1}^{\infty} Q_{M}$. Then, $R \supseteq Q$ and $\zeta \equiv \max _{R} \mu(1)$. It is sufficient to show $R \subseteq Q$. Assume $\mu \in R$ and $\varphi \in C^{\prime}(B)$. Since Linear combinations of the functions $\left\{\varphi_{j}, j=1,2, \ldots\right\}$ are uniformly dense in $C^{\prime}(B)$, there is the Sequence $\left\{\tilde{\varphi}_{k}\right\} \in \operatorname{span}\left\{\varphi_{j}, j=1,2, \ldots\right\}$ such that $\tilde{\varphi}_{k}$ tends to $\varphi$ uniformly as $k \rightarrow \infty$. Hence, $S_{1}, S_{2}$ and $S_{3}$ tend to zero as $k \rightarrow \infty$ where $S_{1}=\sup \left|\varphi_{\xi}-\tilde{\varphi}_{k_{\xi}}\right|$, $S_{2}=\sup \left|\varphi_{t}-\tilde{\varphi}_{k_{t}}\right| \quad$ and $S_{3}=\sup \left|\varphi-\tilde{\varphi}_{k}\right|$. We have $\mu \in R$, and functional $f \rightarrow \mu(f)$ is linear. Therefore, $\mu\left(\tilde{\varphi}_{k}^{g}\right)=\Delta \tilde{\varphi}_{k}$ and

$$
\begin{aligned}
& \left|\mu\left(\varphi^{g}\right)-\Delta \varphi\right|=\left|\mu\left(\varphi^{g}\right)-\Delta \varphi-\mu\left(\tilde{\varphi}_{k}^{g}\right)+\Delta \tilde{\varphi}_{k}\right| \\
& =\mid \int_{\Omega}\left\{\left[\varphi_{\xi}(t, \xi)-\tilde{\varphi}_{k_{\xi}}(t, \xi)\right] g(t, \xi, u)\right. \\
& \left.+\left[\varphi_{t}(t, \xi)-\tilde{\varphi}_{k_{t}}(t, \xi)\right]\right\} d \mu-\left(\Delta \varphi-\Delta \tilde{\varphi}_{k}\right) \mid \\
& \leq S_{1} \int_{\Omega}|g(t, \xi, u)| d \mu+S_{2} \int_{\Omega} d \mu+2 S_{3}
\end{aligned}
$$

Since the right-hand side of the above inequality tends to zero as $k \rightarrow \infty$, while left-hand side is independent of $k$, therefore $\mu\left(\varphi^{g}\right)=\Delta \varphi$. Thus $R \subseteq Q$ and $\zeta \leq \lambda$ which implies $\zeta=\lambda$.

Proposition 4.3.2. The measure $\mu^{*}$ in the set $Q_{M}$ at which the functional $\mu \rightarrow \mu(1)$ attains its maximum has the form

$$
\mu^{*}=\sum_{j=1}^{M+1} \alpha_{j}^{*} \delta\left(z_{j}^{*}\right)
$$

where $\alpha_{j}^{*} \geq 0, z_{j}^{*} \in \Omega$ and $\delta(z)$ is unitary atomic measure with the support being the singleton set $\left\{z_{j}^{*}\right\}$, characterized by $\delta(z)(F)=F(z), z \in \Omega$.

Proof. See appendix of [35].

Therefore, with respect to above descriptions we restrict our attention to finding measure in form $\mu=$ $\sum_{j=1}^{M+1} \alpha_{j} \delta\left(z_{j}\right)$, which maximizes functional $\mu=\mu \rightarrow \mu(1)$ and satisfies in (31) and $M$ number of constraints in the form of (28)-(30). Clearly, $\mu(F)=\sum_{j=1}^{M+1} \alpha_{j} F\left(z_{j}\right), \forall F \in$ $C(\Omega)$. Therefore, by choosing $M_{1}$ number of functions in the form of (32), $S$ number of functions in the form of (34) and $M_{2}^{\prime}$ number of functions in the form of (33), which leads to $M_{2}=6 M_{2}^{\prime}$ number of functions of the type (17) where are numbered sequentially as $\theta_{h}$, $h=1, \ldots, M_{2}$, infinite dimensional problem (27)-(31) is approximated with following finite dimensional nonlinear programming (NLP) problem:

$$
\underset{\alpha_{j} \geq 0, z_{j} \in M^{+}(\Omega)}{\operatorname{Maximize}} \sum_{j=1}^{M+1} \alpha_{j}
$$

Subject to

$$
\begin{aligned}
& \sum_{j=1}^{M+1} \alpha_{j} \varphi_{i}^{g}\left(z_{j}\right)=\Delta \varphi_{i}, i=1, \ldots, M_{1} \\
& \sum_{j=1}^{M+1} \alpha_{j} \theta_{h}\left(z_{j}\right)=0, \quad h=1, \ldots, M_{2} \\
& \sum_{j=1}^{M+1} \alpha_{j} \vartheta_{s}\left(z_{j}\right)=a_{\vartheta}, \quad s=1, \ldots, S \\
& \sum_{j=1}^{M+1} \alpha_{j} H\left(z_{j}\right) \leq \gamma
\end{aligned}
$$

where, $M=M_{1}+M_{2}+S$. We confront with NLP with more than $2(M+1)$ unknowns $\alpha_{j}, z_{j}, j=1, \ldots, M+1$. Finally, the following proposition enables us to approximate the problem via the finite dimensional linear programming problem.

Proposition 4.3.3. Let $\Omega_{N}=\left\{y_{1}, y_{2}, \ldots, y_{N}\right\}$ be a countable dense subset of $\Omega$, for any $N$ sufficiently large number. Given $\varepsilon>0$, a measure $v \in M^{+}(\Omega)$ can be 
found such that:

$$
\begin{aligned}
& \left|v\left(\varphi_{i}^{g}\right)-\mu^{*}\left(\varphi_{i}^{g}\right)\right| \leq \varepsilon \quad i=1, \ldots, M_{1} \\
& \left|v\left(\theta_{h}\right)-\mu^{*}\left(\theta_{h}\right)\right| \leq \varepsilon \quad h=1, \ldots, M_{2} \\
& \left|v\left(\vartheta_{s}\right)-\mu^{*}\left(\vartheta_{s}\right)\right| \leq \varepsilon \quad s=1, \ldots, S \\
& \left|v(H)-\mu^{*}(H)\right| \leq \varepsilon
\end{aligned}
$$

where measure $v$ has the form

$$
v=\sum_{j=1}^{M+1} \alpha_{j}^{*} \delta\left(z_{j}\right)
$$

where the coefficients $\alpha_{j}^{*}, j=1, \ldots, M+1$ are the same as optimal measure (35), and $z_{j} \in \Omega_{N}, j=1, \ldots, M+1$.

Proof. We rename functions $\varphi_{i}^{g}$ 's, $\theta_{h}$ 's, $v_{s}$ 's and $H$ sequentially as $f_{j}, j=1,2, \ldots, M+1$. Then, for $j=1, \ldots$, $M+1$,

$$
\begin{aligned}
& \left|\left(\mu^{*}-v\right) f_{j}\right|=\left|\sum_{i=1}^{M+1} \alpha_{i}^{*}\left[f_{j}\left(z_{i}^{*}\right)-f_{j}\left(z_{i}\right)\right]\right| \\
& \leq\left(\sum_{i=1}^{M+1} \alpha_{i}^{*}\right) \max _{i, j}\left|f_{j}\left(z_{i}^{*}\right)-f_{j}\left(z_{i}\right)\right|
\end{aligned}
$$

$f_{j}$ 's are continues. Therefore, $\max _{i, j}$ can be made less than $\frac{\varepsilon}{\sum_{j=1}^{M+1} \alpha_{j}^{*}}$ by choosing $z_{i}, i=1,2, \ldots, M+1$, sufficiently near to $z_{i}^{*}$.

For construction of dense subset $\Omega_{N}, J$ is divided to $S$ subintervals as follows:

$$
J_{s}=\left[t_{0}+\frac{(s-1) \Delta T}{S-1}, t_{0}+\frac{s \Delta T}{S-1}\right), s=1,2, \ldots, S-1
$$

and

$$
J_{S}=\left[t_{l}, t_{f}\right)
$$

Furthermore, intervals $A_{i}$ 's and $U_{j}$ 's are divided into $n_{i}$ and $m_{j}$ subintervals respectively, then the set $\Omega$ is divided into $N=S n_{1} n_{2} n_{3} n_{4} n_{5} m_{1} m_{2}$ cells. One point is chosen from each cell. In this way we will have a grid of points, which are numbered sequentially as $y_{j}=\left(t_{j}, \xi_{1_{j}}, \ldots\right.$, $\left.\xi_{6_{j}}, u_{P_{j}}, u_{R_{j}}\right), j=1, \ldots, N$.

Remark 2. It is well known that each function type (34) can be approximated in a nice way by a linear combination of characteristic function of subintervals of $J$. In practice we consider $\vartheta_{s}(t)=\chi_{J_{s}}(t), s=1, \ldots, S$ instead of functions of the type (34), where $J_{s}$ 's are given by (46) and $\chi_{J_{s}}$ denotes the characteristic function of $J_{s}$. The main reason for this choice of $\vartheta_{s}$ 's is related to their essential role in construction of control functions. For more details see $[35,36]$.

Considering (45) the NLP (36)-(40) is converted to the following LP:

$$
\underset{\alpha_{j} \geq 0}{\operatorname{Maximize}} \sum_{j=1}^{N} \alpha_{j}
$$

subject to

$$
\begin{gathered}
\sum_{j=1}^{N} \alpha_{j} \varphi_{i}^{g}\left(y_{j}\right)=\Delta \varphi_{i}, \quad i=1, \ldots, M_{1} \\
\sum_{j=1}^{N} \alpha_{j} \theta_{h}\left(y_{j}\right)=0, \quad h=1, \ldots, M_{2} \\
\sum_{j=1}^{N} \alpha_{j} H\left(y_{j}\right) \leq \gamma \\
\sum_{j=1}^{l} \alpha_{j}=\frac{\Delta T}{S-1} \\
\vdots \\
\sum_{j=(S-2) l+1}^{(S-1) l} \alpha_{j}=\frac{\Delta T}{S-1} \\
\sum_{j=(S-1) l+l}^{S l} \alpha_{j}=t_{f}-t_{l} \\
\xi_{i}\left(t_{f}\right) \in A_{i}, i=2,3,4,5,6
\end{gathered}
$$

where $l=\frac{N}{S}$. Of course, we need only to construct the function $u($.$) , since the \xi($.$) is simply the corresponding$ solution of differential Equations (1)-(6) which can be estimated numericall. Using simplex method, nonzero optimal solution $\alpha_{i_{1}}^{*}, \alpha_{i_{2}}^{*}, \ldots, \alpha_{i_{p}}^{*}, i_{1}<i_{2}<\ldots<i_{p}$ of LP (47)-(52) can be found where $p$ cannot exceed the number of constraints i.e., $p \leq M_{1}+M_{2}+S+1$.

Setting $\alpha_{i 0}^{*}=t_{0}$, piecewise control pair $u(t)=\left(u_{P}(t)\right.$, $\left.u_{R}(t)\right)$ which approximate the action of the optimal control, is constructed based on these nonzero coefficients as follows [35,36]:

$$
u(t)=\left\{\begin{array}{cc}
\left(u_{P_{i_{j}}}, u_{R_{i_{j}}}\right) \quad t \in\left[\sum_{h=0}^{j-1} \alpha_{i_{h}}^{*}, \sum_{h=0}^{j} \alpha_{i_{h}}^{*}\right), \quad j=1,2, \ldots, p \\
0 \quad \text { otherewise }
\end{array}\right.
$$

It should be remembered, $u_{P_{i_{j}}}$ and $u_{R_{i_{j}}}$ are respectively 7 th and 8 th components of $y_{i_{j}}$. 


\section{Numerical Results}

In our implementation, we set $M_{1}=14$ and chosen functions $\varphi$ from $C^{\prime}(B)$ are as follows:

$$
\xi_{1}, \xi_{2}, \xi_{3}, \xi_{4}, \xi_{5}, \xi_{6}, t \xi_{1}, \xi_{1} \xi_{2}, \xi_{1} \xi_{3}, \xi_{1} \xi_{4}, \xi_{1} \xi_{5}, \xi_{1} \xi_{6}, \xi_{1}^{2}, t \xi_{1}^{2}
$$

Furthermore, we set $M_{2}^{\prime}=2$. Hence, we have $M_{2}=12$ number of functions in the form of (17). Parameter $S$ is set to 11 and desired lower bound for optimal time is set to $t_{l}=2007.5$ (5.5 years). Setting $u=(0,0)$, and solving ODE (1)-(6) using 4th order Runge-Kutta method, shows that at $t_{0}=620, \xi\left(t_{0}\right)=(350,12.40,1.26,0.16$, 18454) . Starting points of our simulation runs are:

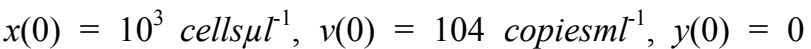

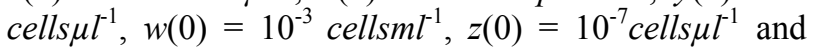
$r(0)=2 \times 10^{-7}$ mlcopies $^{-1}$ day $^{-1}$.

Maximum values for $u_{P}$ and $u_{R}$, are 0.7 and $9 \times 10^{-10}$ respectively [7]. Therefore, the coefficient $\sigma$ for balancing both PI and RTI costs in (8) is set to $\sigma=$ $\left(\frac{0.7}{9 \times 10^{-10}}\right)^{2}$. Furthermore, the total costs are bounded above by $\gamma=480$. By using controllability, considered ranges for states and controls and corresponding partitions for construction of $y_{j}, j=1, \ldots, N$ are summarized in Table 2. Note that, selected values from the set $U_{1}$ for construction of $y_{j}$ 's are $0,0.4$ and 0.7 . These values indicate off, moderate and strong PI-therapy. Similarly, corresponding values for RTI control are $0,5 \times 10^{-10}$ and $9 \times$ $10^{-10}$ [7]. Therefore, we have linear programming with $M=33$ constraints and $N=59405$ unknowns, that is solved using simplex method and environment of MATLAB. Implementing the corresponding LP model, the suboptimal time has been found $t_{f}^{*}=2133.2$ (71.11 Months).

Figure 1 shows the resulting suboptimal control pair. The response of the system to the control functions is depicted in Figure 2. Figure 2(a) shows that condition
(14) violates in a subinterval of $J$, which is due to approximate nature of control pair and can be ignored. Because, the length of this subinterval is very small as compared to the length of $J$. We found $\xi_{1}\left(t_{f}^{*}\right)=199.28$, which is close to exact value i.e., 200. From Figures 2(a) and 2(b), we see drop in the number of CD4+ T-cells, and a rise in viral load following the initial infection until about the third month. After this time, CD4+ T-cells start recovering and virus starts decreasing due to the immune response, but can never eradicate virus completely. Then CD4+ T-cells level decreases and viral load increases due to de struction of immune system in absence of treatment. Figures 2(b) and 2(c) show a clear correlation between the CTLe and virus population. As the virus increases upon initial infection, CTLe increases in order to decrease the virus. Once this is accomplished, virus decreases. Then virus grows back slowly, and this triggers an increase in the CTLe population. CTLe, further increases in an attempt to keep the virus at constant levels but lose the battle because of virus-induced impairment of CD4+ T-cell function, in absence of treatment (dotted line). Memory CTL responses depend on the

Table 2. Considered ranges for states and controls and corresponding number of partitions.

\begin{tabular}{ccc}
\hline State & Range & Number of partitions \\
\hline$\xi_{1}$ & $A_{1}=[200,1000]$ & $n_{1}=5$ \\
$\xi_{2}$ & $A_{2}=[5,30]$ & $n_{2}=3$ \\
$\xi_{3}$ & $A_{3}=[0,1.6]$ & $n_{3}=2$ \\
$\xi_{4}$ & $A_{4}=[0,1.3]$ & $n_{4}=2$ \\
$\xi_{5}$ & $A_{5}=[500,35000]$ & $n_{5}=10$ \\
$\xi_{6}$ & $A_{6}=\left[0,2 \times 10^{-7}\right]$ & $n_{6}=1$ \\
$u_{P}$ & $U_{1}=[0,0.7]$ & $m_{1}=3$ \\
$u_{R}$ & $U_{2}=\left[0,9 \times 10^{-10}\right]$ & $m_{2}=3$ \\
\hline
\end{tabular}
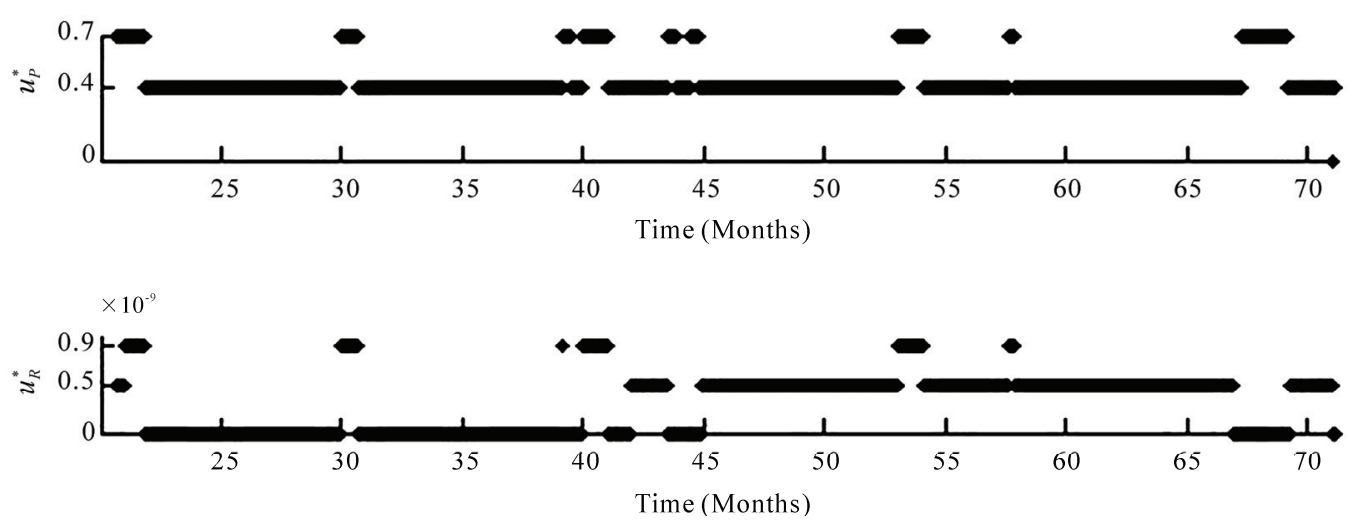

Figure 1. The suboptimal piecewise constant control pair $u_{P}^{*}($.$) and u_{R}^{*}($.$) .$ 




(a)

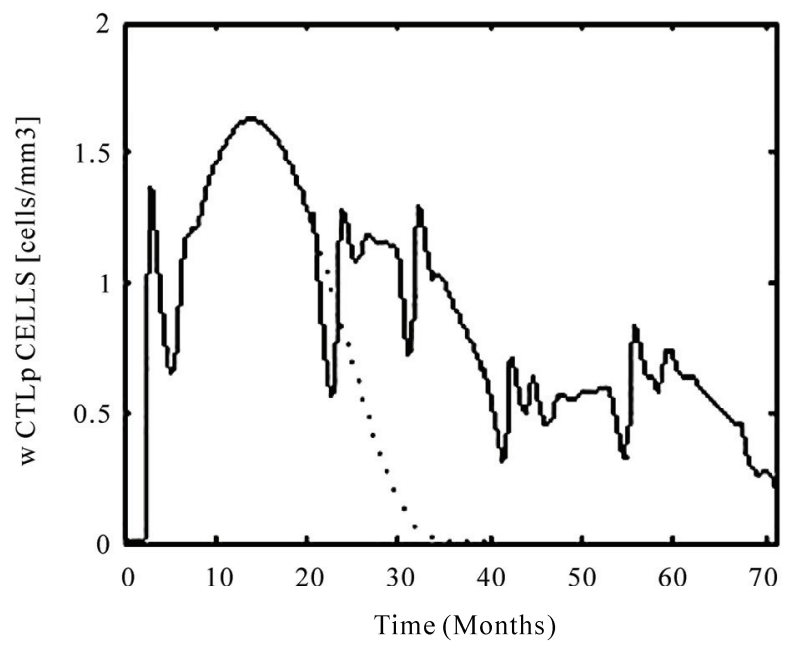

(c)

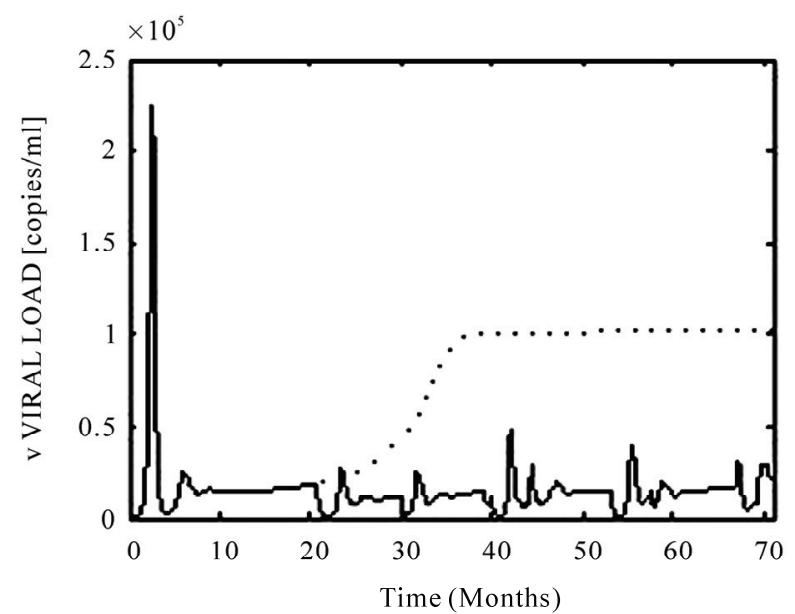

(b)

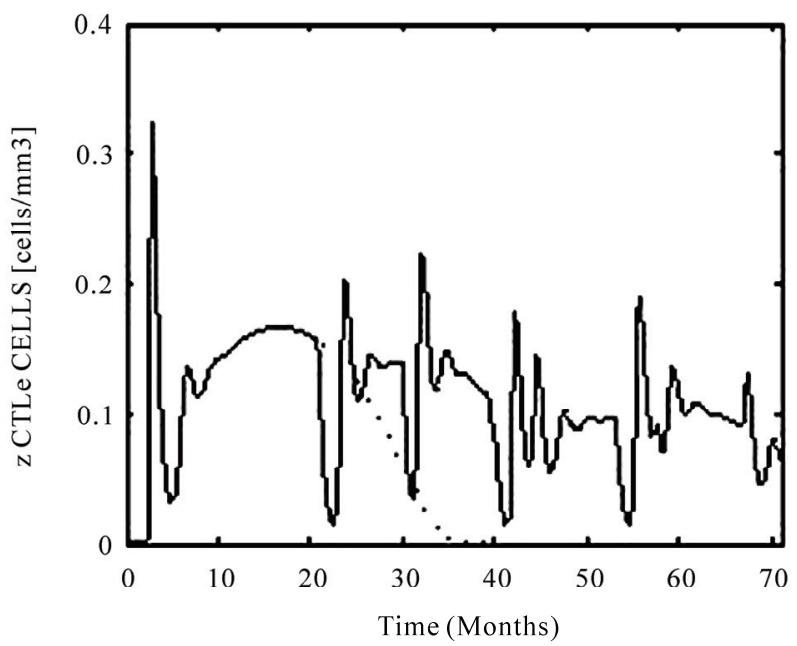

(d)

Figure 2. Dynamic behavior of the state variables $x, v, w$ and $z$ versus time in the case of untreated (dotted line) and treated infected patients (solid line).

presence of CD4+ T-cell help. Figures 2(a) and 2(b) show that, in presence of treatment the virus is controlled to very low levels and CD4+ T-cells are maintained above the critical levels for relatively long time. Therefore, immune response expands for relatively long time successfully. Furthermore, these figures indicate inversecoloration between viral load and CD4+ T-cells level. From Figures 2(c) and 2(d) interestingly, a decrease in CTL's occurs in response to therapy can be observed. The extent of the decrease is directly correlated with the increase in treatment effectiveness which is consistent with experimental findings [37].

\section{Conclusions}

In this paper, we considered a system of ordinary differ- ential equations, which describe various aspects of the interaction of HIV with healthy cells in fast progressive patient, for constructing a time optimal control problem which maximizes asymptomatic stage of patient. A measure theoretical method is used to solve such kind of problems, and numerical results, confirmed the effectiveness of this approach.

\section{References}

[1] T. W. Chun, L. Stuyver, S. B. Mizell, L. A. Ehler, J. A. Mican, M. Baseler, A. L. Lloyd, M. A. Nowak and A. S. Fauci, "Presence of an Inducible HIV-1 Latent Reservoir During Highly Active Antiretroviral Therapy," Proceedings of the National Academy of Sciences, Vol. 94, No. 24, 1997, pp. 13193-13197.

[2] D. Finzi, M. Hermankova, T. Pierson, L. M. Carruth, C. 
Buck, R. E. Chaisson, T. C. Quinn, K. Chadwick, J. Margolick, R. Brookmeyer, et al., "Identification of a Reservoir for HIV-1 in Patients on Highly Active Antiretroviral Therapy," Science, Vol. 278, No. 5341, 1997, pp. $1295-1300$

[3] J. K. Wong, M. Hezareh, H. F. Gunthard, D. V. Havlir, C. C. Ignacio, C. A. Spina and D. D. Richman, "Recovery of Replication-Competent HIV Despite Prolonged Suppression of Plasma Viremia," Science, Vol. 278, No. 5341, 1997, pp. 1291-1295.

[4] M. M. Hadjiandreou, R. Conejeros and V. S. Vassiliadis, "Towards a Long-Term Model Construction for the Dynamic Simulation of HIV Infection," Mathematical Biosciences and Engineering, Vol. 4, No. 3, 2007, pp. 489504.

[5] A. S. Perelson, A. U. Neumann, M. Markowitz, et al., "HIV-1 Dynamics in Vivo: Virion Clearance Rate, Infected Cell Life-Span, and Viral Generation Time," Science, Vol. 271, No. 5255, 1996, pp. 1582-1586.

[6] D. Wodarz and M. A. Nowak, "Specific Therapy Regimes Could Lead to Long-Term Immunological Control of HIV," Proceedings of the National Academy of Sciences, Vol. 96, No. 6, 1999, pp. 14464-14469.

[7] A. Landi, A. Mazzoldi, C. Andreoni, M. Bianchi, A. Cavallini, M. Laurino, L. Ricotti, R. Iuliano, B. Matteoli and L. Ceccherini-Nelli, "Modelling and Control of HIV Dynamics," Computer Methods and Programs in Biomedicine, Vol. 89, No. 2, 2008, pp. 162-168.

[8] K. R. Fister, S. Lenhart and J. S. McNally, "Optimizing Chemotherapy in an HIV Model," Electronic Journal of Differential Equations, Vol. 1998, No. 32, 1998, pp. 112.

[9] M. M. Hadjiandreou, R. Conejeros and D. I. Wilson, "Long-Term HIV Dynamics Subject to Continuous Therapy and Structured Treatment Interruptions," Chemical Engineering Science, Vol. 64, No. 7, 2009, pp. 16001617.

[10] W. Garira, D. S. Musekwa and T. Shiri, "Optimal Control of Combined Therapy in a Single Strain HIV-1 Model," Electronic Journal of Differential Equations, Vol. 2005, No. 52, 2005, pp. 1-22.

[11] J. Karrakchou, M. Rachik and S. Gourari, "Optimal Control and Infectiology: Application to an HIV/AIDS Model," Journal of Applied Mathematics and Computing, Vol. 177, No. 2, 2006, pp. 807-818.

[12] A. Heydari, M. H. Farahi and A. A. Heydari, "Chemotherapy in an HIV Model by a Pair of Optimal Control," Proceedings of the 7th WSEAS International Conference on Simulation, Modelling and Optimization, Beijing, 2007, pp. 58-63.

[13] B. M. Adams, H. T. Banks, H. D. Kwon and H. T. Tran, "Dynamic Multidrug Therapies for HIV: Optimal and STI Control Approaches," Mathematical Biosciences and Engineering, Vol. 1, No. 2, 2004, pp. 223-241.

[14] F. Neri, J. Toivanen and R. A. E. Mäkinen, “An Adaptive Evolutionary Algorithm with Intelligent Mutation Local Searchers for Designing Multidrug Therapies for HIV," Applied Intelligence, Vol. 27, No. 3, 2007, pp. 219-235.
[15] R. Culshaw, S. Ruan and R. J. Spiteri, "Optimal HIV Treatment by Maximizing Immune Response," Journal of Mathematical Biology, Vol. 48, No. 5, 2004, pp. 545-562.

[16] O. Krakovska and L. M. Wahl, "Costs Versus Benefits: Best Possible and Best Practical Treatment Regimens for HIV," Journal of Mathematical Biology, Vol. 54, No. 3, 2007, pp. 385-406.

[17] C. D. Myburgh and K. H. Wong, "An Optimal Control Approach to Therapeutic Intervention in HIV Infected Individuals," Proceedings of the Third International Conference on Control Theory and Applications, Pretoria, South Africa, 2001.

[18] B. M. Adams, H. T. Banks, M. Davidian, H. D. Kwon, H. T. Tran, S. N. Wynne and E. S. Rosenberg, "HIV Dynamics: Modeling, Data Analysis, and Optimal Treatment Protocols," Journal of Computational and Applied Mathematics, Vol. 184, No. 1, 2005, pp. 10-49.

[19] J. Alvarez-Ramirez, M. Meraz and J. X. Velasco-Hernandez, "Feedback Control of the Chemotherapy of HIV," International Journal of Bifurcation and Chaos, Vol. 10, No. 9, 2000, pp. 2207-2219.

[20] S. Butler, D. Kirschner and S. Lenhart, "Optimal Control of Chemotherapy Affecting the Infectivity of HIV," In: O. Arino, D. Axelrod, M. Kimmel and M. Langlais, Eds., Advances in Mathematical Population Dynamics: Molecules, Cells, Man, World Scientific, Singapore, pp.104120.

[21] H. Shim, S. J. Han, C. C. Chung, S. Nam and J. H. Seo, "Optimal Scheduling of Drug Treatment for HIV Infection: Continuous dose Control and Receding Horizon Control," International Journal of Control, Automation, and Systems, Vol. 1, No. 3, 2003, pp. 401-407.

[22] D. Kirschner, S. Lenhart and S. Serbin, "Optimal Control of the Chemotherapy of HIV Infection: Scheduling, Amounts and Initiation of Treatment," Journal of Mathematical Biology, Vol. 35, No. 7, 1997, pp. 775-792.

[23] U. Ledzewicz and H. Schättler, "On Optimal Controls for a General Mathematical Model for Chemotherapy of HIV," Proceedings of the 2002 American Control Conference, Anchorage, Vol. 5, 2002, pp. 3454-3459.

[24] R. Zurakowski, M. J. Messina, S. E. Tuna and A. R. Teel, "HIV Treatment Scheduling Via Robust Nonlinear Model Predictive Control," Proceedings of the 5th Asian Control Conference, Melbourne, Vol. 1, 2004, pp. 25-32.

[25] R. Zurakowski and A. R. Teel, "Enhancing Immune Response to HIV Infection Using MPC-Based Treatment Scheduling," Proceedings of the 2003 American Control Conference, Denver, Vol. 2, 2003, pp. 1182-1187.

[26] R. Zurakowski, A. R. Teel and D. Wodarz, "Utilizing Alternate Target Cells in Treating HIV Infection through Scheduled Treatment Interruptions," Proceedings of the 2004 American Control Conference, Melbourne, Vol. 1, 2004, pp. 946-951.

[27] R. Zurakowski and A. R. Teel, "A Model Predictive Control Based Scheduling Method for HIV Therapy," Journal of Theoretical Biology, Vol. 238, No. 2, 2006, pp. 368-382.

[28] H. T. Banks, H. D. Kwon, J. Toivanen and H. T. Tran, 
"An State Dependent Riccati Equation Based Estimator Approach for HIV Feedback Control," Optimal Control Applications and Methods, Vol. 27, No. 2, 2006, pp. 93121.

[29] M. A. L. Caetano and T. Yoneyama, "Short and Long Period Optimization of Drug Doses in the Treatment of AIDS," Anais da Academia Brasileira de Ciências, Vol. 74, No. 3, 2002, pp. 379-392.

[30] A. M. Jeffrey, X. Xia and I. K. Craig, "When to Initiate HIV Therapy: A Control Theoretic Approach," IEEE Transactions on Biomedical Engineering, Vol. 50, No. 11, 2003, pp. 1213-1220.

[31] J. J. Kutch, P. Gurfil, "Optimal Control of HIV Infection with a Continuously-Mutating Viral Population," Proceedings of the 2002 American Control Conference, Anchorage, Vol. 5, 2002, pp. 4033-4038.

[32] P. Borrow, A. Tishon, S. Lee, J. Xu, I. S. Grewal, M. B. Oldstone and R. A. Flavell, "CD40L-Deficient Mice Show Deficits in Antiviral Immunity and Have an ImPaired Memory CD8 CTL," Journal of Experimental
Medicine, Vol. 183, No. 5, 1996, pp. 2129-2142.

[33] C. Young, "Calculus of Variations and Optimal Control Theory," Sunders, Philadelphia, 1969.

[34] D. A. Wilson and J. E. Rubio, "Existence of Optimal Controls for the Diffusion Equation," Journal of Optimization Theory and Applications, Vol. 22, 1977, pp. 91101.

[35] J. E. Rubio, "Control and Optimization: The Linear Treatment of Non-linear Problems," Manchester University Press, Manchester, 1986.

[36] H. H. Mehne, M. H. Farahi and A. V. Kamyad, "MILP Modelling for the Time Optimal Control Problem in The Case of Multiple Targets," Optimal Control Applications and Methods, Vol. 27, No. 2, 2005, pp. 77-91.

[37] X. Jin, et al., "An Antigenic Threshold for Maintaining Human Immunodeficiency Virus Type 1-Specific Cytotoxic T Lymphocytes," Molecular Medicine, Vol. 6, 2000, pp. 803-809. 